\title{
Evaluation of Cognitive Behaviour Combined with Biofeedback Therapy Based on the Fusion of Normed Space and Banach Space on the Emotional State of Patients with Coronary Artery Bypass Grafting
}

\author{
X. WANG AND Q. YUAN* \\ Tangshan Workers' Hospital, Tangshan, Hebei Province, 063000, China
}

\begin{abstract}
Wang and Yuan: Evaluation of Cognitive Behaviour of Patients with Coronary Artery Bypass Grafting
\end{abstract}
In this paper, the effect of the cognitive behaviour combined with the biofeedback therapy on the evaluation of the emotional status in patients with coronary artery bypass grafting was evaluated. Fifty-two hospitalized patients who are planned to undergo coronary artery bypass grafting were randomly divided into the rehabilitation group and the control group under the normed space and the Banach space. The rehabilitation group was treated by the cognitive behaviour combined with the biofeedback therapy. Compared with the control group, the patients in the rehabilitation group have decreased significantly under the normed space and the Banach space after the operation; while the 6 min walking distance of the patients in the rehabilitation group was increased significantly. The concentration of TNF- $\alpha$ of the patients in the rehabilitation group was significantly decreased and the time of the ventilator-assisted ventilation and the time of intensive care for the patients in the rehabilitation group was shorter than that in the control group. Compared to the preoperative conditions, the left ventricular ejection fraction of the patients in the rehabilitation group was significantly improved. Correlation analysis is carried out on the aforementioned observation indexes for the patients in the rehabilitation group. The results show that the decline in the normed space and the Banach space has significant positive correlation with the extension of the 6 min walking distance, respectively. Cognitive behaviour combined with the biofeedback therapy could improve the anxiety and depression of the patients after undergoing the coronary artery bypass grafting, improve the exercise capacity and promote the recovery of the cardiac function.

Key words: Coronary artery bypass grafting, normed space and Banach space, emotional state, effect evaluation

With the continuous improvement of the living standards and changes in the lifestyle, the incidence of coronary heart disease has also increased and its treatment methods have been quite developed. The position of cardiac rehabilitation in the prevention and treatment of modern cardiovascular diseases has also improved, which has become an important part of the rehabilitation medicine ${ }^{[1,2]}$. It is not only a simple biofeedback therapy, but also including the multi-angle, individualized comprehensive program such as the rehabilitation education, the behavior correction, the cognitive behavior and so on. Coronary artery bypass grafting (CABG) has become an important treatment method for the severe coronary heart diseases ${ }^{[3]}$. Although CABG can directly solve the problem of reperfusion of the stenotic coronary artery, it is a major negative life event because the stress caused by the stress reaction incurred due to the waiting for the operation, the anaesthesia and the trauma often can lead to or even aggravate the feelings of anxiety and depression in patients with coronary heart disease ${ }^{[4,5]}$. Studies have shown that $38 \%$ of the patients who underwent $\mathrm{CABG}$ experienced varying degrees of depression, while those with depression have 2 3 times higher risk of cardiovascular diseases than those without depression ${ }^{[6]}$. The depression and anxiety has an impact on patients with heart disease not only in their social contact, family, work and leisure activities, but also in their re-admission and mortality rates. Anxiety can affect the cardiac output by increasing the heart

*Address for correspondence

E-mail: yuanq@gmail.com 
rate, thereby shortening the duration of the diastolic period and reducing the coronary perfusion ${ }^{[7,8]}$. At the same time, during the waiting for the operation and 3 to $4 \mathrm{w}$ after the operation, majority of the patients choose to reduce exercise and rest in bed as they are concerned of increasing the cardiac burden, which will inevitably lead to the cardiovascular dysfunction; while the trauma that comes from the operation itself can also severely reduce the physical strength of the patients ${ }^{[9]}$.

At present, the cognitive behaviours of the patients who undergo CABG include routine care, educational explanation, behavioural therapy, cognitive therapy, biofeedback therapy, supportive therapy, group therapy and so on. In addition, the effects of some of the new intervention measures such as the visual imaging therapy, tai chi, music therapy, hypnosis and so on are still in the study ${ }^{[10,11]}$. The most widely used cognitive behaviours at home and abroad are the combined application of a number of methods that are aimed at individual differences. The biofeedback therapy and the cognitive therapy are adopted for the cognitive behaviour. At the same time, rehabilitation education is carried out on the patients who undergo CABG, so that the patients can understand the bypass graft surgery correctly and relieve the self-imagination and postoperative fear (for example, the fear caused by the intensive care, fear that coughing, sneezing and so on might cause the disruption of the incision, worrying about the postoperative quality of survival and the decline of work ability, doubts about the postoperative incision pain, swelling of the lower extremities and so on) $)^{[12-14]}$.

Therefore, it can be seen that there is a mutual influence relationship between the exercise capability and the cognitive behaviour of the patients who undergo CABG. The combination of the cognitive behaviour and the biofeedback therapy can play an important role in the rehabilitation of the patients after the operation. The morbidity rate of the coronary atherosclerotic heart disease has increased year after year, which has become a major disease that threaten the human health ${ }^{[15]}$. With the continuous development of treatment means such as the thrombolysis, coronary stenting, CABG and so on, scholars at home and abroad have shifted their focus to the rehabilitation of the coronary heart disease. Studies in other countries have suggested that the cognitive behaviour combined with the biofeedback therapy can greatly improve the onset and performance of the coronary heart disease. Over the past $30 \mathrm{y}$, foreign countries have accumulated a lot of experience in the cardiac rehabilitation medicine centered on the early activities and psychotherapy. Significant progress has been made in reducing the mortality rate and the disability rate as well. In this study, the targeted cognitive behaviour combined with biofeedback therapy was carried out to improve the therapeutic effect of the patients who underwent CABG.

\section{MATERIALS AND METHODS}

\section{Research subjects and grouping:}

A total of 52 patients who underwent CABG in the Department of Cardiology, First Affiliated Hospital of China Medical University from June to November 2009 were selected. The coronary artery angiography verified the presence of the lesion in the anterior descending branch and the left circumflex branch of the left coronary artery as well as the right coronary artery in all the patients, with severe stenosis of lumen $>75 \%$, who were critical patients with the cardiac function grade II-IV, obesity associated with diabetes, hypertension, valvular disease and so on. Among them, 28 patients (17 patients in the rehabilitation group and 11 patients in the control group) had myocardial infarction, and the rest were patients with angina pectoris. The echocardiography showed cardiac enlargement to different degrees. Inclusion criteria were, postoperative hemodynamic stability; no angina pectoris attacks; no acute myocarditis or pericarditis; relatively good blood glucose control; no peripheral vascular thrombosis or embolism; no cerebrovascular accident; no postoperative incision poor healing; no new ECG ischemic changes; no history of chronic mental illness; with normal cognitive function, able to comply with the rehabilitation treatment, and all the patients signed the informed consent.

The 52 patients were randomly divided into the rehabilitation group $(\mathrm{n}=27)$ and the control group $(n=25)$. The general information of the patients in the two groups are as shown in Table 1. The patients in both the groups received the CABG preoperative and postoperative drugs such as calcium antagonists, ACE inhibitors and beta blockers. The rehabilitation treatment method described in 1.2 was adopted for the patients in the rehabilitation group, while the patients in the control group received only routine treatment.

\section{Rehabilitation treatment, biofeedback therapy:}

Achieving the level 11 13 in the 15 ratings of perceived exertion (Borg rating) was the best when the patients carried out exercise. The exercise time was 10 40 min, 
TABLE 1: COMPARISON OF THE CLINICAL DATA OF PATIENTS OF THE TWO GROUPS

\begin{tabular}{|c|c|c|}
\hline Parameters & $\begin{array}{c}\text { The } \\
\text { rehabilitation } \\
\text { group }\end{array}$ & $\begin{array}{l}\text { The control } \\
\text { group }\end{array}$ \\
\hline Gender (male/female) & $18 / 9$ & $18 / 7$ \\
\hline Age $(y)$ & $62.31 \pm 7.89$ & $61.88 \pm 8.24$ \\
\hline Body mass index $\left(\mathrm{kg} / \mathrm{m}^{2}\right)$ & $25.08 \pm 2.10$ & $25.67 \pm 1.70$ \\
\hline $\begin{array}{l}\text { Associated with diabetes } \\
\text { (case) }\end{array}$ & 8 & 6 \\
\hline $\begin{array}{l}\text { Associated with } \\
\text { hypertension (case) }\end{array}$ & 18 & 20 \\
\hline $\begin{array}{l}\text { Associated with } \\
\text { diabetes+hypertension } \\
\text { (case) }\end{array}$ & 7 & 5 \\
\hline Smoking (case) & 12 & 10 \\
\hline Addicted to drinking (case) & 1 & 1 \\
\hline $\begin{array}{l}\text { Both smoking and addicted } \\
\text { to drinking }\end{array}$ & 1 & 1 \\
\hline Cardiopulmonary bypass & 9 & 8 \\
\hline $\begin{array}{l}\text { Without cardiopulmonary } \\
\text { bypass }\end{array}$ & 18 & 17 \\
\hline Cardiac function grade II-III & 25 & 23 \\
\hline Cardiac function grade IV & 2 & 2 \\
\hline
\end{tabular}

with an average of $30 \mathrm{~min}$, not including the warm-up and the finishing exercise time (3 5 min) before and after each training. The exercise intensity was gradually increased in accordance to the Borg rating and the walking distance on a daily basis. Walking in the aerobic exercises was adopted as the main form of exercise. When the patients could keep walking for 10 15 min (up to $600 \mathrm{~m}$ ), they could receive the stairs climbing training with support. The heart rate and blood pressure of the patients were monitored before and after the exercise rehabilitation, and the respiratory rate, the change in the facial expression and so on of the patients were closely observed during the walking process. With reference to Pollock's thoracic heart surgery hospitalization rehabilitation method and the domestic coronary heart disease rehabilitation programs, combined with the recent foreign cardiac rehabilitation progress, a set of comprehensive rehabilitation treatment program was adopted in this experiment.

\section{Cognitive behaviour:}

After admission, cognitive therapy and progressive biofeedback therapy were used to address the anxiety and depression of the patients. Through the cognitive therapy, cognitive reconstruction, psychological coping, problem solving and other means were adopted to reconstruct the cognitive structure of the patients, change the misconceptions and bad behaviours of patients on the coronary heart disease, the operation and the cardiac rehabilitation, and answer their questions for the diseases, the postoperative and rehabilitation processes. The biofeedback therapy lasted 10 20 min each time. The basic requirement was as the following: in a quiet environment, the patient was lying flat, adopting the abdominal, deep and slow breathing, staying focused, and consciously feeling the tension and relaxation of the main muscle groups. The cognitive therapy adopted the cognitive reconstruction, psychological coping, problem solving and other methods to reconstruct the cognitive structure of the patients, thus improving the state of the depression and anxiety feelings of the patients. Biofeedback therapy and cognitive therapy were adopted in this experiment in accordance with the foreign literature, and these two therapies were continuously applied to all aspects of the rehabilitation in the whole process. The overall rehabilitation procedures were shown in Table 2 as the following.

\section{Observation indexes and evaluation methods:}

The surgery was performed by the cardiac surgeon, and all patients received similar preoperative preparation, general anaesthesia, extubating, intensive care unit monitoring, determination of the tumour necrosis factor- $\alpha$ (TNF- $\alpha)$, echocardiography, the normed space and the Banach space and 6 min walking test.

The main evaluation basis for the normed space was the frequency of the occurrence of the symptoms defined by the item, which could be divided into 4 levels, A indicated never or very seldom, B indicated some of the time, $\mathrm{C}$ indicated quite a lot of time, and $\mathrm{D}$ indicated most or all of the time, which was rated as 1, 2, 3 and 4 points, respectively. In the scoring, the 5th, 9 th, 13 th, 17 th and 19th item must be scored in a reverse manner. For example, in item 9, if a patient felt calm of spirit and even of temper, and could easily sit quietly, A was rated, $\mathrm{B}$ was rated 3 points, $\mathrm{C}$ was rated 2 points and $\mathrm{D}$ was rated 1 point. The scores of the 20 items each were added to obtain the rough score, which was then multiplied by 1.25 and then the integer was rounded off to obtain the standard score. The reference value for the normal upper limit of the total rough score was 40 points, and the reference value for the upper limit of the standard score was 50. The higher the score was, the more obvious the anxiety tendency was. The calculation method in the Banach space was the same as that of the normed space. 


\begin{tabular}{|c|c|c|}
\hline & Content of the exercise & Cognitive behavior \\
\hline Preop & $\begin{array}{l}6 \text { min walking distance test; Instructing the } \\
\text { patients to practice the abdominal-pursed lip } \\
\text { breathing; effective cough training (including } \\
\text { explosive coughing and coughing in a whisper) }\end{array}$ & $\begin{array}{l}\text { Self-assessment of the depression and anxiety; } \\
\text { Guide the patients to carry out the biofeedback } \\
\text { therapy; introduce the coronary artery bypass } \\
\text { grafting and the situation in the postoperative } \\
\text { care unit to the patients; answer all kinds of } \\
\text { doubts of the patients to relieve the fear of the } \\
\text { patients before the operation and after they wake } \\
\text { up from the anesthesia }\end{array}$ \\
\hline
\end{tabular}

Postop

\begin{tabular}{|c|c|}
\hline Day $1 \sim 3$ & $\begin{array}{c}\text { After the condition was stable, the patients } \\
\text { started active movement of the wrists, ankle joints } \\
\text { in bed as well as the knee flexion and knee joint } \\
\text { movement in bed. }\end{array}$ \\
\hline Day 4 5 & $\begin{array}{c}\text { Start bedside activities, go to the toilet and taking } \\
\text { walk properly }\end{array}$ \\
\hline Day $5 \sim 6$ & $\begin{array}{l}\text { Walking } 100 \sim 300 \mathrm{~m} \text { (in accordance with the } \\
\text { patient's respectively condition) }\end{array}$ \\
\hline Day $7 \sim 14$ & $\begin{array}{c}\text { Determine the walking distance in accordance with } \\
\text { the patient's own condition on a daily basis, and } \\
\text { carry out the stair climbing exercise properly with } \\
\text { support. }\end{array}$ \\
\hline Day 15 & 6 min walking distance test \\
\hline $\begin{array}{l}\text { Day } 16 \text { } \\
\text { Discharge }\end{array}$ & Walking \\
\hline
\end{tabular}

Inform the patients of the operation situation and the current status; relieve the psychological stress of the patients; tell the patients the necessity and the precautions of the rehabilitation exercise

Provide education on the postoperative diet considerations and rehabilitation

Introduce the knowledge related to the coronary heart disease and the effect of the rehabilitation; encourage the patients to communicate with those with good rehabilitation effect so as to improve the confidence and enthusiasm of the patients for rehabilitation; teach the patients to count their pulse by themselves and introduce the perceived exertion to the patients

Answer the questions that the patients have encountered in their postoperative life, eliminate the concerns and fears of the patients about the life outside the hospital; continue to carry out the rehabilitation education

Self-assessment of the depression and anxiety

Education on the discharged and work with the patients to develop a discharge rehabilitation program together
The fasting venous blood samples were collected from all the patients in the early morning, placed in the anticoagulation tube containing sodium citrate, mixed thoroughly and then centrifuged on the whole blood (3000 rpm, $4^{\circ}, 10 \mathrm{~min}$ ). The supernatant was taken and stored in a refrigerator at the low temperature of $-70^{\circ}$ for later test. The concentration of TNF- $\alpha$ was determined by the enzyme linked-immuno-sorbent assay in a uniform manner. The kit was purchased from Wuhan Boster Biological Technology Co., Ltd., and the various operation steps were performed strictly in accordance with the instructions of the kit. Echocardiography was performed by the same physician, and the uniform examination was performed using the Vivid7, USA. The left ventricular ejection fraction (LVEF) was observed.

The distance of $30 \mathrm{~m}$ was measured and taken in the middle of a quiet, well-ventilated, temperature-friendly corridor of the cardiac surgery ward. A chair was placed at each end for the rest of the patients. The patients were instructed to walk back and forth at a consciously appropriate speed before walking. When they were walking, the patients should walk as fast as possible along the straight line, avoiding the circular route, and the 6 min walking distance (6-MWD) was taken as the test result. If the patients presented fatigue, dizziness, angina pectoris, difficulty in breathing, cold sweat and pale face within $6 \mathrm{~min}$, the test was suspended. The blood pressure, heart rate and respiratory rate of the patients were monitored before and after the test. Rescue drugs such as nitroglycerin were kept available during the test. The postoperative intubation time, intensive care time and hospital stay of the patients in the two groups were statistically analysed. Correlation analysis was carried out on the measured 6-MWD with the LVEF in the normed space and the Banach space, respectively. Statistical analysis on the data was carried out using the SPSS13.0 software package. All the measurement data were expressed as mean \pm standard 
deviation. Statistical analysis was carried out using the $\mathrm{t}$ test, and the correlation analysis of the 6-MWD with the LVEF and Hamilton Depression and Anxiety Scale was carried out, respectively.

\section{RESULTS AND DISCUSSION}

On comparison of the degree of depression and anxiety it was found that there was no difference in the scores of the patients between the 2 groups before the operation. Compared to the score before surgery, the patients in the rehabilitation group showed a significant decrease in the normed space and the Banach space on the $\mathrm{d} 15$ after the operation $(\mathrm{p}<0.01)$. In the comparison between the groups, the scores of various items of the patients in the rehabilitation group post-surgery were all significantly lower than those in the control group $(p<0.01)$. There was a significant difference in the difference of the scale before and after the operation of the patients between the two groups $(\mathrm{p}<0.01)$, as shown in Table 3.

There was no significant difference in the LVEF of the patients between the two groups before the operation. The LVEF level of the patients in the rehabilitation group after the operation was significantly improved compared to that before the operation; while there was no significant difference in the patients of the control group before operation compared to that after the operation. There was no significant difference in the LVEF of the patients between the two groups after the operation. There was no significant difference in the difference of the LVEF of the patients before and after the operation between the two groups ( $p>0.01)$, as shown in Table 3.

There was no significant difference in the 6-MWD between the two groups before the operation ( $\mathrm{p}>0.01)$.
Compared to the 6-MWD before the operation, the 6-MWD of the patients in the rehabilitation group was significantly extended on d 15 after the operation; while the 6-MWD of the patients in the control group showed no significant change $(p>0.01)$. Compared with the patients in the control group, the difference in the 6-MWD of the patients in the rehabilitation group before and after the operation significant, as shown in Table 3.

There was no significant difference in the TNF- $\alpha$ of the patients in the two groups before the operation. The level of TNF- $\alpha$ in the rehabilitation group was significantly lower after the operation than that before the operation and the difference was statistically significant. The TNF- $\alpha$ level of the patients in the control group after the operation was not significantly different from that before the operation. There was a significant difference in the TNF- $\alpha$ levels of the patients between the two groups after the operation $(p<0.01)$, as shown in Table 3. There was a significant difference in the mean intubation time and the intensive care time of the patients between the two groups, as shown in Table 4.

The correlation analysis of 6-MWD in the normed space and the Banach space was carried out, respectively. The results showed that the difference of the 6-MWD before and after the operation (d-6MWD) and the difference before and after the operation in the normed space and the Banach space was positively correlated, respectively $(\mathrm{rA}=0.46, \mathrm{p}<0.01 ; \mathrm{rB}=0.48, \mathrm{p}<0.01)$, as shown in fig. 1 .

As the cardiovascular conditions after the $\mathrm{CABG}$ have been significantly improved, exercise rehabilitation is mostly focused on the training of the cardiovascular function. In order to ensure the safety of the exercise, $6 \mathrm{MWT}$ is especially suitable for the evaluation of the

TABLE 3: COMPARISON OF THE PREOPERATIVE AND POSTOPERATIVE PARAMETERS OF PATIENTS

\begin{tabular}{lcccc}
\hline \multirow{2}{*}{ Item } & \multicolumn{2}{c}{ The control group } & \multicolumn{2}{c}{ The rehabilitation group } \\
\cline { 2 - 5 } & Preop & Postop & Preop & Postop \\
\hline Normed space (score) & $32.24 \pm 4.18$ & $32.24 \pm 4.60$ & $31.85 \pm 4.81$ & $28.00 \pm 3.22$ \\
Banach space (score) & $31.92 \pm 5.15$ & $32.88 \pm 7.06$ & $32.44 \pm 6.92$ & $28.04 \pm 3.18$ \\
LVEF & $54.48 \pm 10.22$ & $56.24 \pm 6.84$ & $53.33 \pm 9.49$ & $59.00 \pm 7.35$ \\
6-MWD $(\mathrm{m})$ & $245.6 \pm 107.07$ & $220.8 \pm 54.25$ & $241.11 \pm 95.46$ & $298.15 \pm 59.24$ \\
TNF-a $(\mathrm{pg} / \mathrm{ml})$ & $48.72 \pm 14.61$ & $50.25 \pm 16.78$ & $49.91 \pm 27.33$ & $42.22 \pm 13.99$ \\
\hline
\end{tabular}

Comparison of the preoperative and postoperative depression, anxiety scale, left ventricular ejection, 6 min walking distance and TNF-a score of the patients between the two groups (mean \pm standard deviation)

TABLE 4: COMPARISON OF THE POSTOPERATIVE INTUBATION TIME AND THE INTENSIVE CARE TIME OF THE PATIENTS BETWEEN THE TWO GROUPS

\begin{tabular}{lcc}
\hline Item & The control group & The rehabilitation group \\
\hline Intubation time & $49.54 \pm 53.13$ & $24.24 \pm 29.61$ \\
Intensive care time & $92.96 \pm 49.18$ & $66.56 \pm 27.09$ \\
\hline
\end{tabular}




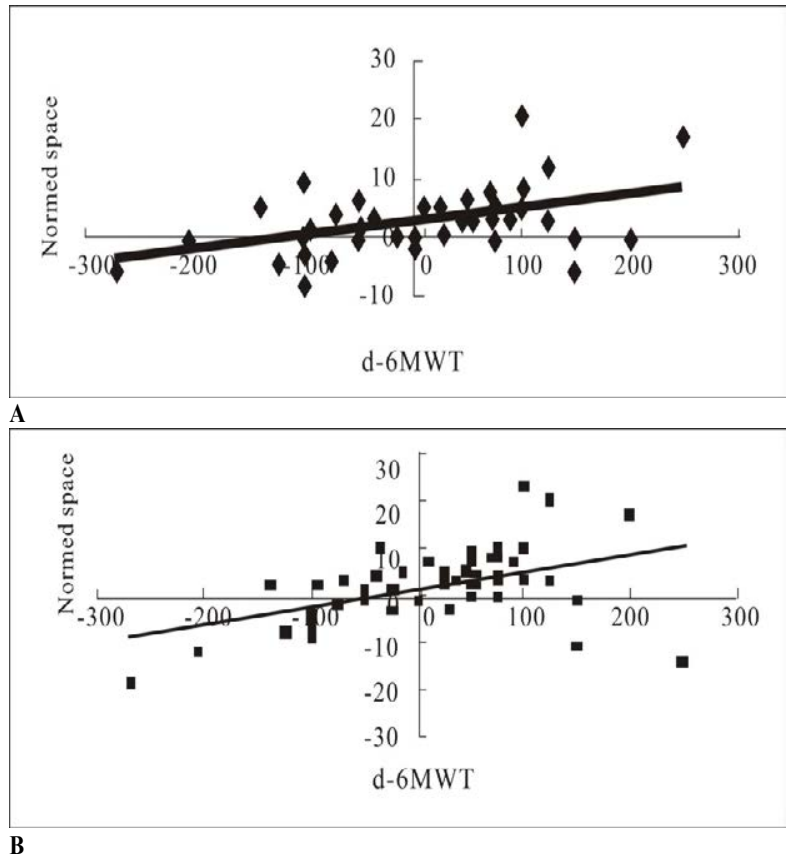

Fig. 1: Scatter plots

(A) D-6MWD in the normed space, (B) D-6MWD in the Banach space

physical activity ability of the patients suffering from the heart disease with low cardiac function and low cardiac storage. In the study, walking training, stair climbing training and other low and medium intensity aerobic exercises were adopted on the basis of the physiological indexes of the patients such as the grading of the cardiac function, the degree of the coronary artery lesion, the rating of perceived exertion, the heart rate, the blood pressure and so on. The results showed that 6-MWD was measured on the d 15 after the surgery, and the 6-MWD of the patients in the rehabilitation group was significantly higher than that in the control group. The LVEF of the patients in the rehabilitation group was higher than that in the control group, and the difference was statistically significant $(p<0.01)$. As the research on the aetiology of coronary heart diseases was carried out in recent years, the important role of TNF- $\alpha$ in the occurrence and the development of the coronary heart disease has been recognized. TNF- $\alpha$ can be affected by a number of factors, such as infection, stress and so on. It was found in the study that 29 patients after undergoing the coronary stenting carried out exercise rehabilitation that lasted $6 \mathrm{w}$. Compared with the control group, the inflammatory markers such as the TNF- $\alpha$ and the C-reactive protein (CRP) were significantly reduced. In the patients with cardiovascular disease and cancer, depression had significant positive correlation with the inflammatory markers CRP, IL-1 and IL-6. In this study, the concentration of TNF- $\alpha$ of the patients in the rehabilitation group after the operation was lower than that before the operation, and the difference was statistically significant; the difference was statistically significance as well when it was compared with that in the control group $(\mathrm{p}<0.01)$. It could be seen that the comprehensive cardiac rehabilitation also had certain effect on the postoperative fluid factors of the patients after undergoing the $\mathrm{CABG}$, which could delay the development of the coronary heart diseases from the level of the body fluid to a certain extent. However, TNF- $\alpha$ had no correlation with the other indexes. After analysis, the reason may be due to the fact that there were many factors that could affect TNF- $\alpha$. The sample size of this experiment was relatively small, and the intervention time was excessively short. Hence, TNF- $\alpha$ showed no obvious correlation with the other indexes. In this study, it was found that the extension of 6MWD of the patients in the CABG rehabilitation group after the operation had significant positive correlation with the decrease in the degree of depression and anxiety. Studies have shown that depression and anxiety can significantly affect the compliance of the patients in the rehabilitation training. Good cognitive behaviour can allow the patients to better comply with the treatment and the training, improve their enthusiasm, and thus improve the training effect. Studies have shown that appropriate biofeedback therapy can improve the degree of depression and anxiety by regulating the endorphins, monoamines, hypothalamic-pituitaryadrenal axis, neurotrophies, nerve conduction pathways and other means. At the same time, the improvement of the exercise capability itself can also alleviate the feelings of the anxiety and anxiety of the patients. As a part of the clinical rehabilitation, the effectiveness of the cardiac rehabilitation has been proven by a number of studies. This study showed that the cognitive behaviour and biofeedback therapy before and after the CABG has played a positive role in a number of aspects such as shortening the extubating and intensive care time, reducing the degree of depression and anxiety, improving the exercise capacity, enhancing the cardiac function, delaying the development of the coronary heart disease and so on. Medical personnel should combine the exercise capability and the psychological status of the patients to develop a scientific and reasonable rehabilitation program before and after the $\mathrm{CABG}$ and carry out the targeted psychological guidance while assisting the patients to restore their exercise capability at the same time, so that the patients can return to their family and the society as soon as possible. 


\section{REFERENCES}

1. Yang JH, Choi SH, Song YB, Hahn JY, Choi JH, Jeong DS, et al. Long-term outcomes of drug-eluting stent implantation versus coronary artery bypass grafting for patients with coronary artery disease and chronic left ventricular systolic dysfunction. Am J Cardiol 2013;112(5):623-9.

2. Angeloni E, Paneni F, Landmesser U, Benedetto U, Melina G, Lüscher TF, et al. Lack of protective role of hdl-c in patients with coronary artery disease undergoing elective coronary artery bypass grafting. Eur Heart J 2013;34(46):3557-62.

3. Yang JH, Choi SH, Song YB, Hahn JY, Choi JH, Dong SJ. Iconography: long-term outcomes of drug-eluting stent implantation versus coronary artery bypass grafting for patients with coronary artery disease and chronic left ventricular systolic dysfunction. Circulation J 2013;77(7):1742-9.

4. Chen SW, Chang $\mathrm{CH}$, Lin YS, Wu VC, Chen DY, Tsai FC. Effect of dialysis dependence and duration on post-coronary artery bypass grafting outcomes in patients with chronic kidney disease: a nationwide cohort study in asia. Int J Cardiol 2016;223:65-71.

5. Mizuno T, Egi K, Sakai K, Oi K, Hachimaru T, Makita T. Minimally circulatory-assisted on-pump beating coronary artery bypass grafting for patients with complex conditions for off-pump surgery. Artifl Organs 2017;41(3):233-41.

6. Hansson EC, Jidéus L, Åberg B, Bjursten H, Dreifaldt M, Holmgren. Coronary artery bypass grafting-related bleeding complications in patients treated with ticagrelor or clopidogrel: a nationwide study. Eur Heart J 2016;37(2):189-97.

7. Ronaldson A, Kidd T, Poole L, Leigh E, Jahangiri M, Steptoe A. Diurnal cortisol rhythm is associated with adverse cardiac events and mortality in coronary artery bypass patients. J Clin Endocrinol Metab 2015;100(10):3676-82.

8. Hassan H, Chatterjee S, Ahmed M, Tripathi B, Pallazo A, Herzog E. All-cause mortality benefit in patients undergoing mitral valve surgery with coronary artery bypass as compared to isolated coronary artery bypass surgery in patients with moderate ischemic mitral regurgitation: a meta-analysis. J Am College Cardiol 2016;67(13):332-7.

9. Rigattieri S, Sciahbasi A, Brilakis ES, Burzotta F, Rathore
S, Pugliese FR. Meta-analysis of radial versus femoral artery approach for coronary procedures in patients with previous coronary artery bypass grafting. Am J Cardiol 2016;117(8):1248-55.

10. Nagendran J, Bozso SJ, Norris CM, Mcalister FA, Appoo JJ, Moon MC. Coronary artery bypass surgery improves outcomes in patients with diabetes and left ventricular dysfunction. J Am College Cardiol 2018;71(8):819-27.

11. Du J, Fan C, Jiang H, Sun H, Li X, Zhao M. Biofeedback combined with cue-exposure as a treatment for heroin addicts. Physiol Behavior 2014;130(6):34-39.

12. Muradregadas SM, Regadas FS, Bezerra CC, de Oliveira MT, Regadas Filho FS, Rodrigues LV. Use of biofeedback combined with diet for treatment of obstructed defecation associated with paradoxical puborectalis contraction (anismus): predictive factors and short-term outcome. Dis Colon Rectum 2016;59(2):115-21.

13. Nishida H, Uchikawa S, Chikazawa G, Kurihara H, Kihara $\mathrm{S}$, Uwabe K. Coronary artery bypass grafting in 105 patients with hemodialysis-dependent renal failure. Artif Organs 2015;25(4):268-72.

14. Ellis SG. Coronary revascularization for patients with diabetes: updated data favor coronary artery bypass grafting. J Am College Cardiol 2013;61(8):817-9.

15. Curl K, Lebude B, Ruggiero N, Fischman D, Rose A, Patel S. Frequency of use of statins and aspirin in patients with previous coronary artery bypass grafting. Am J Cardiol 2016;118(1):4043.

This is an open access article distributed under the terms of the Creative Commons Attribution-NonCommercial-ShareAlike 3.0 License, which allows others to remix, tweak, and build upon the work non-commercially, as long as the author is credited and the new creations are licensed under the identical terms

This article was originally published in a special issue: Special issue on "Drug Development and Human Health in China"

Indian J Pharm Sci 2020:82(1)spl issue2;7-13 